\title{
Satellitendaten in der Geländeklimatologie - Resultate des Projektes «Heat Capacity Mapping Mission» (HCMM)
}

\section{Geländeklimatologische Fragestellungen}

Der hohe Stellenwert von Satellitendaten im Bereich der synoptischen und globalen meteorologischen Datenbeschaffung ist kaum mehr bestritten (WMO, 1977). Mit der technischen Vervollkommnung aller Teilbereiche (Satelliten, Sensoren, Datenübertragung und -verarbeitung) ist auch die Zahl der erfaßbaren meteorologischen Parameter, z.T. dreidimensional und zeitlich praktisch kontinuierlich, weiter angestiegen. Probleme grundsätzlicher Art ergeben sich daraus, daß fernerkundete Daten ausschließlich auf der Interpretation radiometrischer Meßwerte beruhen und deshalb nicht ohne weiteres mit den "klassischen", meist im direkten Kontakt mit der Atmosphäre gewonnenen Daten vergleichbar sind. Als Beispiel sei hier etwa die vertikale Luftemperaturverteilung der freien Atmosphäre erwähnt, die vom Satelliten mit Hilfe multispektraler Radiometerdaten auf Grund der Strahlungsabsorptions- und -emissionseigenschaften der atmosphärischen Gase berechnet wird - gegenüber der traditionellen Kontaktmessung entlang des Radiosondageprofils.

Im Gegensatz zum synoptischen Maßstab spielen Satellitendaten im Bereich der Geländeklimatologie eine noch eher untergeordnete Rolle. Dies rührt einerseits daher, daß das Auflösungsvermögen der Satellitensensoren bezogen auf die räumliche und zeitliche Variabilität vieler Geländeklimaparameter nicht genügt. Zum andern lassen sich aus technischphysikalischen Gründen die Meßwerte der nur geringmächtigen planetaren Grenzschicht nicht ohne weiteres von den Einflüssen der Erdoberfläche sowie der übrigen Atmosphäre trennen. $\mathrm{Da} \beta$ ungeachtet dieser prinzipiellen Schwierigkeiten ein erfolgversprechender Einsatz von Satellitendaten im Rahmen ausgewählter geländeklimatischer Fragestellungen möglich ist, soll in den folgenden Ausführungen skizziert werden.

Topographie und Bodenbeschaffenheit beeinflussen den Energie- und Strahlungshaushalt sowie die Durchlüftungsverhältnisse nachhaltig - zwei Themenkreise, die heute im Zentrum vieler Forschungsbemühungen stehen (WANNER, 1982), deren Bearbeitung aber gleichzeitig auch mit einem sehr hohen meßtechnischen Aufwand verbunden sein kann (CLIMOD, 1981). Die Verknüpfung einer überblickbaren Anzahl von Stationsmessungen, gezielter Meßfahrten und flächen- deckender Flugzeug- oder Satellitendaten dürfte aber in vielen Fällen eine optimale Methodenkombination ergeben - auch mit Blick auf Prozeßanalysen und Modellstudien. Fig. 1 versucht, die Stellung von Satellitendaten in bezug auf konkrete geländeklimatische Schwerpunktthemen und die Verknüpfung mit anderen Datenquellen schematisch darzustellen. Wenn wir dabei von den Schlüsselparametern in Durchlüftungsstudien ausgehen - dem räumlich stark modifizierten Windfeld, dem vertikal bei Inversionslagen stark eingeschränkten Luftmassenaustausch, ferner dem unterschiedlichen Wärmeverhalten der verschiedenen Oberflächentypen -, dann liegt in den bereits heute verfügbaren Satellitendaten ein noch nicht vollständig ausgeschöpftes Informationspotential.

Die Bestimmung der in Fig. 1 enthaltenen Schlüsselgrößen erfolgt direkt oder über Indikatoren:

1. Oberflächentemperatur: direkte Bestimmung aus Thermal-Infrarot-Aufnahmen, teilweise unter Ausschaltung atmosphärischer Effekte (Kap. 5).

2. Inversionshöhen:

- direkt mit Hilfe der Nebelmeerhöhe

- indirekt aus der (vor allem nächtlichen) Bodenoberflächentemperaturverteilung.

3. Windfeld: Strömungsfelder mit Hilfe von Tracern (Rauchfahnen), Wolkenformationen (Cu-Straßen, Leewellen), Wolkenstrukturen (Wellen in der Nebelmeeroberfläche).

Wie die einzelnen Stichworte vermuten lassen, sind unter Umständen bereits mit einfachem Interpretationsaufwand gute Resultate herleitbar. Häufig aber namentlich bei der pixelgenauen Analyse von Oberflächentemperaturen - lassen sich aufwendige Bildentzerrungen, Datenkalibrierung und Elimination der atmosphärischen Einflüsse nicht umgehen. Zudem ist noch kein Satellitensystem verfügbar, das alle oben aufgeführten Parameter mit gleicher Genauigkeit erfaßt, wie die folgende Zusammenstellung zeigt. (Tab. 1):

Im experimentellen Rahmen haben sich LANDSAT für Nebel- und Strömungsanalysen, NOAA für klimatologische Erhebungen (Nebel, Wolken, Dunst,

Matthias Winiger, Gerrit Nejedly, Zdena Schwab Geographisches Institut der Universität Bern, Hallerstraße 12, 3012 Bern 
Tabelle 1 Charakteristika einiger für Anwendungen in der Geländeklimatologie geeigneter Satelliten (div. Quellen)

\begin{tabular}{|c|c|c|c|c|c|c|c|}
\hline \multirow[b]{2}{*}{ Satellit } & \multirow[b]{2}{*}{ Status } & \multirow[b]{2}{*}{ Flughöhe } & \multirow{2}{*}{$\begin{array}{l}\text { Bedek- } \\
\text { kungs- } \\
\text { zyklus }\end{array}$} & \multirow{2}{*}{$\begin{array}{l}\text { Spektral- } \\
\text { bereiche }\end{array}$} & \multicolumn{2}{|c|}{ Auflösung } & \multirow[t]{2}{*}{ Anwendung } \\
\hline & & & & & räumlich & thermal & \\
\hline LANDSAT-1, 2 & Experimentell & ca. $920 \mathrm{~km}$ & 18 Tage & $\begin{array}{l}0,5-0,6 \mu \mathrm{m} \\
0,6-0,7 \\
0,7-0,8 \\
0,8-1,1\end{array}$ & $80 \mathrm{~m}$ & - & $\begin{array}{l}\text { Nebel, Wolken, } \\
\text { evtl. Dunst } \\
\text { Wind } \\
\text { Schnee }\end{array}$ \\
\hline \multirow[t]{2}{*}{ LANDSAT-3 } & \multirow[t]{2}{*}{ Experimentell } & \multirow[t]{2}{*}{ ca. $920 \mathrm{~km}$} & \multirow[t]{2}{*}{18 Tage } & \begin{tabular}{|l} 
wie oben \\
$0,5-0,7 \mu \mathrm{m}$
\end{tabular} & $\begin{array}{l}80 \mathrm{~m} \\
38 \mathrm{~m}\end{array}$ & - & $\begin{array}{l}\text { wie oben } \\
\text { nur sw-Bilder, }\end{array}$ \\
\hline & & & & $10,4-12,6 \mu \mathrm{m}$ & $250 \mathrm{~m}$ & - & System-Ausfall \\
\hline \multirow[t]{2}{*}{ HCMM } & \multirow[t]{2}{*}{ Experimentell } & \multirow[t]{2}{*}{ ca. $620 \mathrm{~km}$} & \multirow[t]{2}{*}{ (5)-16 Tage } & $0,55-1,1 \mu \mathrm{m}$ & $500 \mathrm{~m}$ & & $\begin{array}{l}\text { Nebel, Wolken, Schnee, } \\
\text { Wind }\end{array}$ \\
\hline & & & & $10,5-12,5 \mu \mathrm{m}$ & $600 \mathrm{~m}$ & $0,4^{\circ} \mathrm{K}$ & $\begin{array}{l}\text { Oberflächentemperaturen } \\
\text { (Wärmeinseln, Kälteseen) }\end{array}$ \\
\hline \multirow[t]{2}{*}{ NOAA $-6,7$} & \multirow[t]{2}{*}{ Operationell } & \multirow[t]{2}{*}{ ca. $830 \mathrm{~km}$} & \multirow[t]{2}{*}{$\begin{array}{l}\text { 4-6mal } \\
\text { pro Tag }\end{array}$} & $\begin{array}{l}0,58-0,68 \mu \mathrm{m} \\
0,725-1,10\end{array}$ & ca. $1 \mathrm{~km}$ & - & $\begin{array}{l}\text { Nebel, Dunst, Wolken } \\
\text { (evtl. Wind) }\end{array}$ \\
\hline & & & & $\begin{array}{l}3,55-3,93 \\
10,5-11,5 \\
11,5-12,5\end{array}$ & ca. $1 \mathrm{~km}$ & ca. $0,15^{\circ} \mathrm{K}$ & $\begin{array}{l}\text { Wolken } \\
\text { Wärmeinseln } \\
\text { (Schnee) }\end{array}$ \\
\hline
\end{tabular}

Anmerkungen:

1) Alle Satelliten decken Perioden von je etwa 2-5 Jahren ab, die sich gegenseitig nicht oder nur teilweise überlappen.

2) Das räumliche Auflösungsvermögen gilt für den Subsatellitenpunkt.

Wärmeinseln) mit gutem Erfolg einsetzen lassen, während HCMM zusätzlich für thermische Analysen (Inversionen) geeignet ist. Die in den folgenden Abschnitten aufgeführten Beispiele sind weitgehend im Rahmen des Projektes HCMM erarbeitet worden.

\section{Die «Heat Capacity Mapping Mission» (HCMM)}

HCMM war ein experimentelles Satellitenprogramm der NASA, welches in erster Linie der Untersuchung des Wärmeverhaltens (Heat Capacity) der Erdoberfläche diente. Thermische Eigenschaften spielen eine Rolle bei agrometeorologischen Fragestellungen (Bodenfeuchtigkeit), vor allem aber auch in der Geologie und Prospektion. Überflüge zum Zeitpunkt größter Bodenerwärmung bzw. stärkster nächtlicher Auskühlung können zwar nicht mit einem einzigen Satelliten realisiert werden, die in Tab. 1 aufgeführten Bahn- und Sensoreigenschaften ergaben aber doch einen sehr brauchbaren Kompromiss.

Sowohl die gute räumliche Auflösung wie die Überflugszeiten - 21/2 Stunden nach Mitternacht bzw. 11/2 Stunden nach Sonnenhöchststand - versprachen auch für geländeklimatologische Untersuchungen optimale Daten. Ein der NASA eingereichtes Proposal mit dem Titel «Topoclimatological and snowhydrological survey of Switzerland» (wINIGER, 1975) wurde angenommen. Drei Themenbereiche standen im Zentrum der vorgeschlagenen Untersuchungen:

1. Welcher Stellenwert kann der Auswertung von Satellitendaten im Rahmen geländeklimatologischer Arbeiten beigemessen werden?

2. Welche Möglichkeiten erschließen analoge und digitale Bildanalyse?

3. Können aus den Strahlungstemperaturen der Erdoberfläche (im folgenden meist Oberflächentemperatur genannt) Kaltluftgebiete abgegrenzt werden?

Der Verifikation der Satellitenmeßwerte bzw. der Verknüpfung mit konventionellen Klimadaten diente ein aufwendiges Flugzeug- und Bodenmeßprogramm. Die Programmschwerpunkte und ihre Verknüpfung sind in Fig. 2 zusammenfassend dargestellt. Wesentliche Beiträge lieferten zwei Diplomarbeiten (NEJEDLY, 1980; sCH $\mathrm{AB}$, 1981), entscheidende Impulse ergaben sich aber auch aus der Mitarbeit im Projekt CLIMOD 


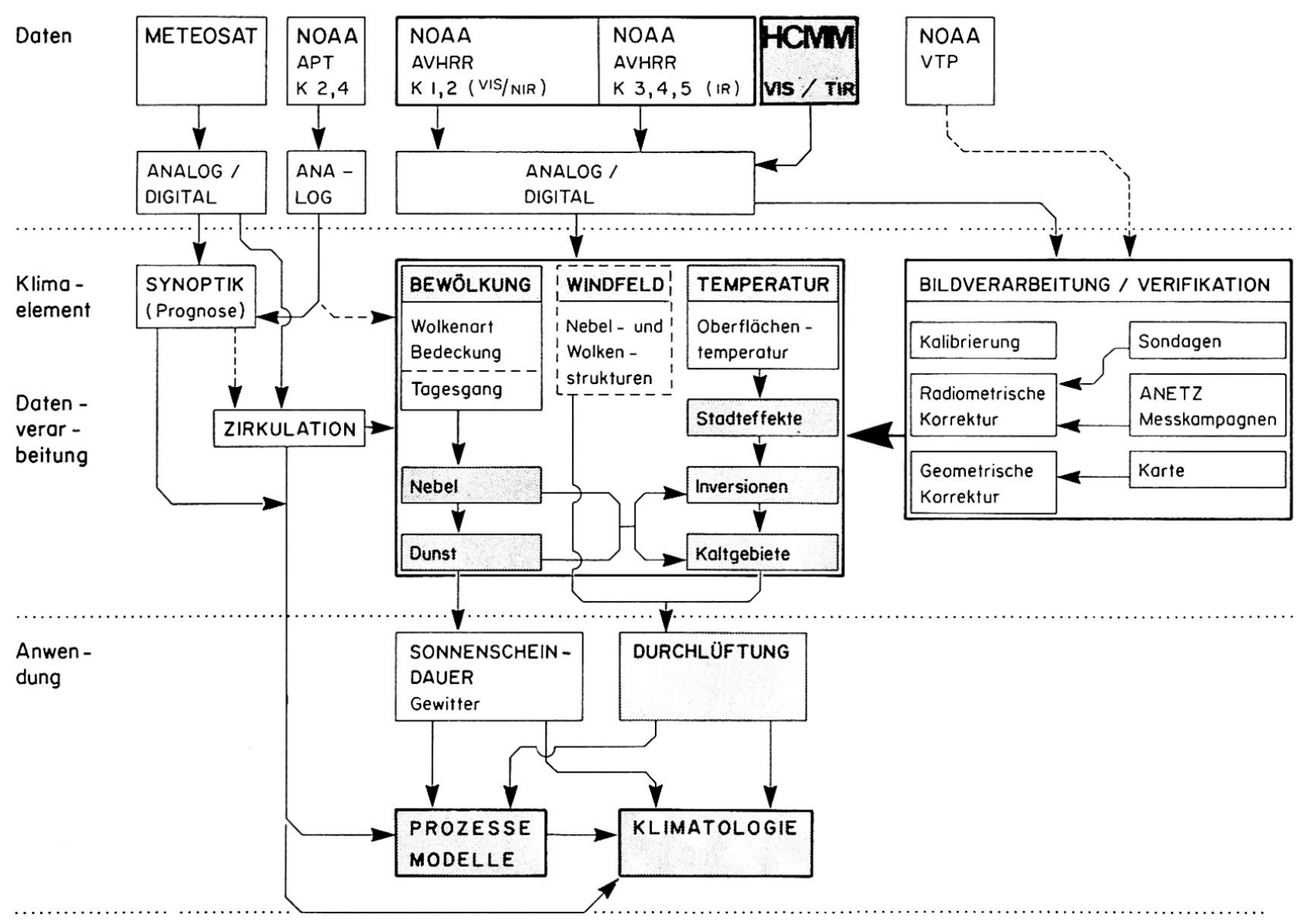

Fig.1: Zur Anwendung von Satellitendaten in der Geländeklimatologie: direkte oder indirekte Bestimmung einzelner Klimaparameter, Datenverarbeitungs- und Verifikationsschwerpunkte.

(CLIMOD, 1981; wINIGER, 1982a). Die wesentlichsten Resultate wurden schließlich in einem zusammenfassenden Schlußbericht der NASA vorgelegt (WINIGER, 1982b).

In den folgenden Abschnitten kann nur auf einzelne Aspekte der HCMM-Datenauswertung eingetreten werden. Insbesondere verzichten wir auf die Darstellung der geometrischen Probleme, die sich nicht grundsätzlich von denjenigen der LANDSAT-Auswertungen unterscheiden (LICHTENEGGER, 1980). Die Möglichkeiten der Wind- und Nebelanalysen werden nur gestreift, da sie an anderer Stelle ausführlicher dargelegt worden sind.

\section{Windanalysen}

Eine direkte Windmessung (Richtung, Windweg) ist mit den verfügbaren Satellitendaten nicht möglich. Immerhin kann bei vorhandener Bewölkung aus deren Anordnung bzw. aus den Strukturen der Wolkenoberfläche das Windfeld qualitativ beurteilt werden. Der ausgeprägte Einfluß der Topographie erzeugt namentlich auf tiefliegenden Nebeldecken ein vielfältiges Muster von Wellen und Längsstrukturen, aus denen mit rel. großer Sicherheit auf das Strömungsfeld geschlossen werden kann und in erster Näherung gut durchlüftete Bereiche von solchen mit stagnierenden Luftmassen unterscheidbar sind. Als Beispiel einer solchen Stromlinienanalyse ist Fig. 3 aufgeführt, die den für die Fragestellung der Studie CLIMOD bedeutungsvollen Kaltluftstrom aus dem Mittelland ins Hochrheintal (winterliche Inversionslagen) räumlich festlegt. Zahlreiche Feldversuche mit Hilfe driftender Schwebeballone bestätigten die Richtigkeit der Satellitenbildauswertungen (CLIMOD, 1981: $85 \mathrm{ff}$.$) .$

\section{Nebelkartierung}

Die Kartierung von Nebelfeldern gehört sicher zu den geländeklimatisch überzeugendsten Auswertungsbeispielen von Satellitenbildern, da sie mit vergleichsweise einfachen methodischen Mitteln und auch bei Satellitendaten etwas geringerer Bodenauflösung (z. B. NOAA) überzeugende Ergebnisse liefern (WANNER, KUNZ, 1982; wINIGER, 1974). Immerhin gilt aber auch 


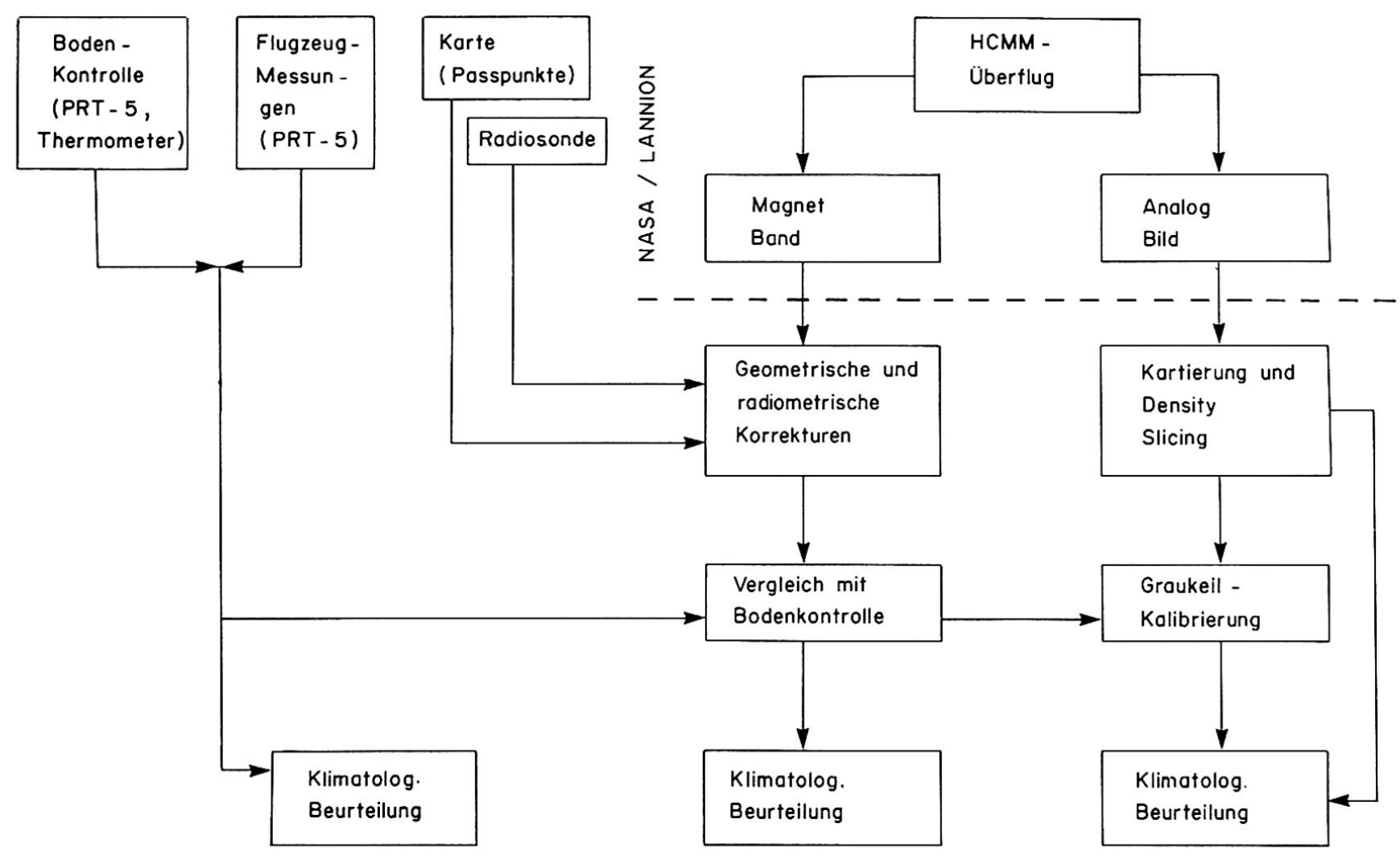

Fig. 2: Auswertungsschema im Rahmen des HCMM-Projektes: analoge und digitale Bildverarbeitung, Verifikation im Gelände und klimatologische Auswertung.

hier zu berücksichtigen, da $\beta$ aus zwei Gründen Abweichungen zu entsprechenden terrestrischen Auswertungen zu erwarten sind: 1 . Die stationsbezogene Nebelbeobachtung beruht auf dem Sichtweitenkriterium (Nebel, wenn Sichtweite $<1 \mathrm{~km}$ ). 2. Die Nebelkartierung mit Hilfe von Satellitenbildern ist natürlich nur möglich, wenn keine Wolkenfelder die Sicht auf die Nebeldecke verhüllen. Beide Einschränkungen ändern aber nichts an der Tatsache, daß die relative, wetterlagenbezogene räumliche Verteilung der Nebelschicht wesentlich differenzierter erfaßbar ist, wenn Satellitenbilder zur Verfügung stehen. Dies ist ein wesentlicher Aspekt bei lufthygienischen Anwendungen, wenn wir davon ausgehen, daß mit der Nebelgrenze die Inversionsschicht festgelegt ist, oder bei der Erstellung von Sonnenenergie-Katastern.

\section{Oberflächentemperaturen}

Die im Spektralbereich 10,5-12,5 $\mu \mathrm{m}$ empfangene thermische Infrarotstrahlung der Satelliten HCMM und NOAA liefern über Kontinenten ein räumlich außerordentlich stark variierendes Bild der Oberflächentemperaturverteilung. Bereits die rein visuelle Interpretation von Wärmebildern zeigt deutlich, daß einerseits die Oberflächenbeschaffenheit (Wasser, Wald, Agrargebiete, Städte, Wüsten), zum andern aber die Topographie (Höhenlage, Exposition, Gelän- deform) je nach Tages- und Jahreszeit das Temperaturfeld verschieden stark beeinflussen. Da aber auch weitere Parameter, wie Bodenfeuchtigkeit, Wind und Strahlungsverhältnisse (letztlich also die Wetterlagen), ebenfalls auf die Oberflächentemperaturverteilung

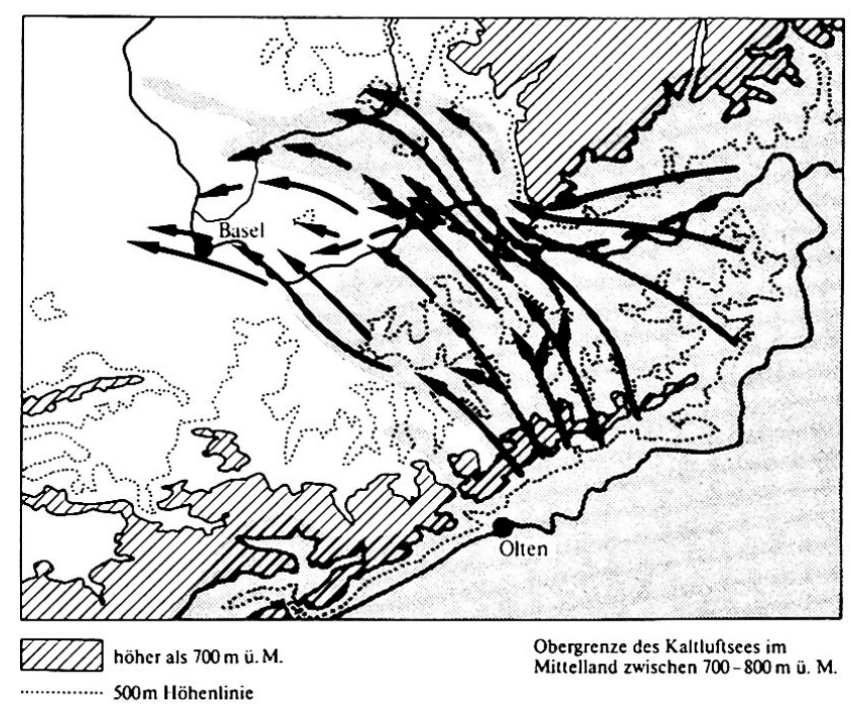

Fig. 3: Windfeldanalysen aus Wolkenstrukturen. Beispiel der Strömung im Hochrheintal, wie sie sich aus der Nebelverteilung und den Oberflächenstrukturen in der Nebeldecke bestimmen läßt (LANDSAT-, HCMM-, NOAA-Daten). Das Strömungsfeld ist abhängig von der Mächtigkeit des aus dem Mittelland ausfließenden Kaltluftsees (WINIGER, 1982a). 
Tabelle 2 Vergleich zwischen atmosphärisch korrigierten HCMM-Oberflächentemperaturmessungen und Bodenkontrollen (Angaben in ${ }^{\circ} \mathrm{C}$ ). Aus NEJEDLY, 1980: 77

\begin{tabular}{|c|c|c|c|c|c|c|}
\hline $\begin{array}{l}\text { Datum } \\
\text { Zeit }\end{array}$ & Testgebiete & $T_{B}$ & $T_{B-k o r r}$ & $\mathrm{~T}_{\text {HCMM-korr }}$ & \multicolumn{2}{|c|}{ Fehler } \\
\hline $\begin{array}{l}\text { 31.8.1978 } \\
\text { 12.55 Uhr MEZ }\end{array}$ & $\begin{array}{l}\text { Murtensee } \\
\text { Schmelzender Schnee (Aletsch) } \\
\text { Seeland (Landwirtschaftsflächen) }\end{array}$ & $\begin{array}{r}+21,5 \\
0,0 \\
+32,5\end{array}$ & $\begin{array}{r}+19,2 \\
0,0 \\
+28,9\end{array}$ & $\begin{array}{l}+13,5 \\
-\quad 4,5 \\
+20,8\end{array}$ & $\left.\begin{array}{l}-5,7 \\
-4,5 \\
-7,8\end{array}\right)$ & $-6,0$ \\
\hline $\begin{array}{l}\text { 16.5.1979 } \\
\text { 01.37 Uhr MEZ }\end{array}$ & $\begin{array}{l}\text { Murtensee } \\
\text { Brienzersee } \\
\text { Seeland (Landwirtschaftsflächen) }\end{array}$ & $\begin{array}{l}+15,0 \\
+11,0 \\
+9,5\end{array}$ & $\begin{array}{r}+13,8 \\
+10,4 \\
+9,2\end{array}$ & $\begin{array}{l}+5,1 \\
+2,0 \\
+\quad 0,5\end{array}$ & $\begin{array}{l}-8,7 \\
-8,4 \\
-8,5\end{array}$ & $-8,6$ \\
\hline $\begin{array}{l}\text { 3.6.1978 } \\
\text { 02.49 Uhr MEZ }\end{array}$ & Brienzersee & $+12,8$ & $+12,2$ & $+12,0$ & $-0,2$ & \\
\hline
\end{tabular}

$\mathrm{T}_{\mathrm{B}}$ : Radiometrische oder thermometrische Kontrollmessung am Boden (teilweise aus dem Flugzeug).

$\mathrm{T}_{\mathrm{B} \text {-korr }}$ : Kontrollmeßwert, wie er nach Berücksichtigung des atmosphärischen Einflusses vom Satelliten gemessen werden sollte (= Hypothetischer Wert).

$\mathrm{T}_{\text {HCMM-korr }}$ : Bodentemperaturwert, wie er vom Satelliten HCMM unter Berücksichtigung des atmosphärischen Einflusses tatsächlich gemessen worden ist.

Fehler: Differenz zwischen $\mathrm{T}_{\mathrm{HCMM}}$-korr und $\mathrm{T}_{\mathrm{B} \text {-korr }}$

einwirken, ist angedeutet, welch komplexe Verflechtung von Randbedingungen letztlich einen bestimmten Temperaturwert bedingen. Trotzdem lassen sich bestimmte Temperaturstrukturen in den SatellitenThermalbildern mit großer Regelmäßigkeit ausmachen: kalte Talsohlen und deutliche Temperatursprünge in höheren Lagen (in Nachtaufnahmen); erhöhte Temperaturen in Siedlungsbereichen (in Tagaufnahmen). Die Untersuchung des Zusammenhangs zwischen diesen thermischen Charakteristika und den bekannten geländeklimatischen Erscheinungen nächtlicher Kaltluftansammlungen in Tallagen, Lufttemperaturinversionen und städtischen Wärmeinseln liegt auf der Hand und bildete denn auch einen Schwerpunkt unserer Arbeiten. Dabei waren folgende Fragen abzuklären: 1. Wie beeinflussen die Atmosphäre und die variable Emissivität des Bodens die radiometrische, vom Satelliten empfangene Information? 2. Lassen sich die beiden Haupteinflußgrößen "Topographie» und «Bodenbedeckung» bei den zu untersuchenden windschwachen Hochdrucklagen voneinander trennen? 3. Besteht ein Zusammenhang zwischen Bodenoberflächentemperatur und der Temperatur der darüberliegenden Luftschicht?

\subsection{Atmosphärische Einflüsse}

Absorption und Emission von Strahlung in der Atmosphäre sind im Empfindlichkeitsbereich der Satellitensensoren $(10,5-12,5 \mu \mathrm{m})$ vor allem abhängig vom vertikalen Luftfeuchtigkeits- und Temperaturprofil. Mit Hilfe des von der NASA zur Verfügung gestellten und von uns modifizierten RATRA (Strahlungstransfer-)Modells, sowie unter Verwendung von Radiosondage-Werten lassen sich die Einflüsse der Atmosphäre auf die Satellitenmessung berechnen. Dabei werden in der Regel Temperaturunterschiede der Erdoberfläche durch die darüberliegende Luftschicht ausgeglichen. Allfällige Korrekturen werden dabei umso größer ausfallen, je stärker Boden- und Lufttemperatur voneinander abweichen und je bedeutender die Temperaturintervalle auf der Erdoberfläche selber sind. Umgekehrt können für hochgelegene

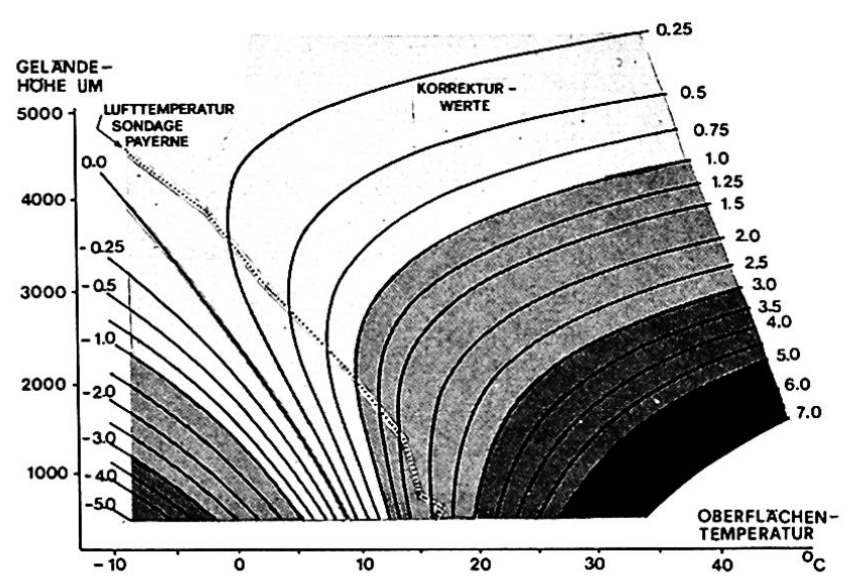

Fig. 4 Korrekturwerte zur Elimination des atmosphärischen Einflusses, berechnet mit den Radiosondenwerten (Payerne) vom 16.8.1978, 01 Uhr MEZ. Der Korrekturbetrag ist abhängig von der Bodenoberflächentemperatur und der Geländehöhe. Beispiel: eine in $2000 \mathrm{~m}$ ü. M. gemessene Oberflächentemperatur von $+20^{\circ} \mathrm{C}$ wird im Satelliten um $2,5^{\circ} \mathrm{C}$ zu tief gemessen, muß also um diesen Betrag korrigiert werden. 
Gebiete die Einflüsse der Atmosphäre meist vernachlässigt werden, da die Hauptmasse des absorbierenden bzw. emittierenden Wasserdampfes in der untersten Troposphäre konzentriert ist. Als Beispiel zeigt Fig. 4 die für einen bestimmten Aufnahmezeitpunkt berechneten Korrekturwerte, als Funktion der auftretenden Oberflächentemperaturwerte und der Geländehöhe. Dabei wird nochmals deutlich, daß Korrekturen für Gebiete oberhalb $3000 \mathrm{~m}$ ü. M. praktisch wegfallen. In tiefern Lagen dürften sie bei Nachtaufnahmen max. $\pm 1,5^{\circ} \mathrm{C}$, am Tag dagegen teilweise mehr als $\pm 3^{\circ} \mathrm{C}$ ausmachen, je nach den vorkommenden Bodentemperaturen und immer bezogen auf den dem Diagramm zugrunde liegenden atmosphärischen $\mathrm{Zu}$ stand.

Obwohl die Emissivitäten unterschiedlicher Materialien stark voneinander abweichen, ist beim integrierenden Effekt des beschränkten räumlichen Auflösungsvermögens der Satellitensensoren die vereinfachende Annahme zulässig, eine einheitliche terrestrische Emissivität von 0,97 allfälligen Korrekturen zugrunde zu legen. Daraus resultiert eine Strahlungstemperatur, die um $2-4^{\circ} \mathrm{C}$ tiefer liegt als die reale Oberflächentemperatur. In den meisten Fällen blieb allerdings diese Korrektur unberücksichtigt, da es um qualitative Temperaturfeldbegrenzungen (Kälteseen) oder relative Unterschiede (Wärmeinseln) ging.

Ein Vergleich atmosphärisch korrigierter Strahlungstemperaturen mit simultan aus Flugzeugen oder am Boden gemessenen Werten ist in Tab. 2 zusammengestellt.

Erstaunlich ist die fast ideale Übereinstimmung von Satellitenwert und Bodenkontrolle für die Aufnahme vom 3.6.78, während für die rel. großen Abweichungen der beiden andern Kontrolltage keine befriedigende Erklärung gefunden werden konnte. Wahrscheinlich ist sie aber in der Kalibrierung des Radiometers zu suchen, die von der NASA mindestens einmal geändert worden ist. Vergleichsmessungen anderer Projektgruppen liegen innerhalb der gleichen Streubreite wie unsere Werte (REINIGER, 1981).

\subsection{Einflüsse von Topographie und Oberflächen- bedeckung}

Die Modifikation des Temperaturfeldes durch die unterschiedlichen Bodenbedeckungskategorien konnte bereits aus den Radiometermessungen im Rahmen des Projektes CLIMOD detailliert bestimmt werden. Dabei treten die markantesten Unterschiede im Vergleich von Tag- und Nachtmessungen zwischen den Kategorien «Stadt» und "Landwirtschaftsgebiete» (Äcker und Wiesen) auf, während andrerseits "Wald» und vor allem natürlich "Wasserflächen» ausgleichende Wirkung zeigen (wINIGER, in HAEFNER et al., 1980: 72). Zugleich weisen aber alle Bedeckungsklassen auch einen deutlichen, tages- und jahreszeitlich sowie von der Exposition modifizierten Höhengradienten auf.

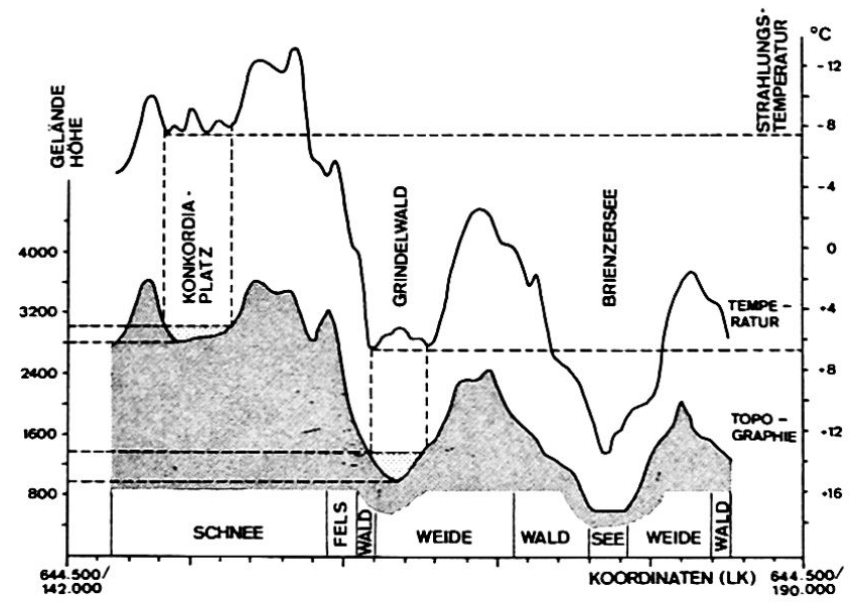

Fig. 5: Gelände- und Oberflächentemperaturprofil vom Konkordiaplatz zum Brienzersee entlang der LK-Koordinate 644.500 für den 3.6.1978, 01.50 Uhr MEZ. Die Temperaturwerte sind aus digitalen HCMM-Daten berechnet worden (ohne atmosphärische Korrektur). Punktiert sind Temperaturinversionen (Kaltluftseen) im Talkessel von Grindelwald und auf dem Konkordiaplatz hervorgehoben (SCHWAB, 1981: 115).

Fig. 5 zeigt ein Oberflächentemperatur-Profil durch das Berner Oberland, basierend auf den HCMMDigitaldaten vom 3.6.78, 02.37 Uhr MEZ. Die Höhenabhängigkeit der Temperatur, gemittelt über alle Oberflächenkategorien und Expositionen, kann bereits dem Kurvenverlauf entnommen werden. Aus den Daten läßt sich ein Temperaturgradient bestimmen, der mit $-0,8^{\circ} \mathrm{C} / 100 \mathrm{~m}$ angenähert dem Höhengradienten der freien Atmosphäre (Sondage Payerne) entspricht, welcher $1 \frac{1}{2}$ Stunden vor dem Satellitenüberflugstermin $-0,7^{\circ} \mathrm{C} / 100 \mathrm{~m}$ beträgt. Die Lufttemperatur der freien Atmosphäre liegt dabei durchwegs $5-6^{\circ} \mathrm{C}$ über derjenigen der Bodenoberfläche. Im Temperaturprofil der Fig. 5 zeichnen sich aber auch deutlich zwei Ausnahmen ab: die Plateau- bzw. Muldenlage von Konkordiaplatz und Grindelwald. In beiden Fällen handelt es sich um deutliche Temperaturinversionen, die ihre Entsprechung in einer Lufttemperaturinversion (Kaltluftsee) finden (vgl. auch COENDET, 1979). Solche Kaltzonen lassen sich bezeichnenderweise mit großer Regelmäßigkeit in ähnlichen topographischen Situationen in wetterlagenabhängiger Ausprägung finden. Dabei sind nicht nur Bodeninversionen, sondern auch langandauernde Höheninversionen räumlich abgrenzbar, in günstigen Fällen z.T. auf $50-100 \mathrm{~m}$ genau bezüglich ihrer Höhenlage (vgl. WINIGER, in HAEFNER et al., 1980: 72).

Die Kartierung der relativen Temperaturverteilung auf der Grundlage analoger Bilddaten ist für einzelne Gebietsausschnitte und zwei Überflugsdaten in Fig. 6 dargestellt. Die Beispiele vom 16.11 .78 sind Ausschnitte aus einer gesamtschweizerischen Kartierung (NEJEDLY, in WINIGER, 1982b). Dabei zeigt sich nochmals der streng topographische Bezug der lokalen Kältezonen beim Vergleich der beiden Aus- 


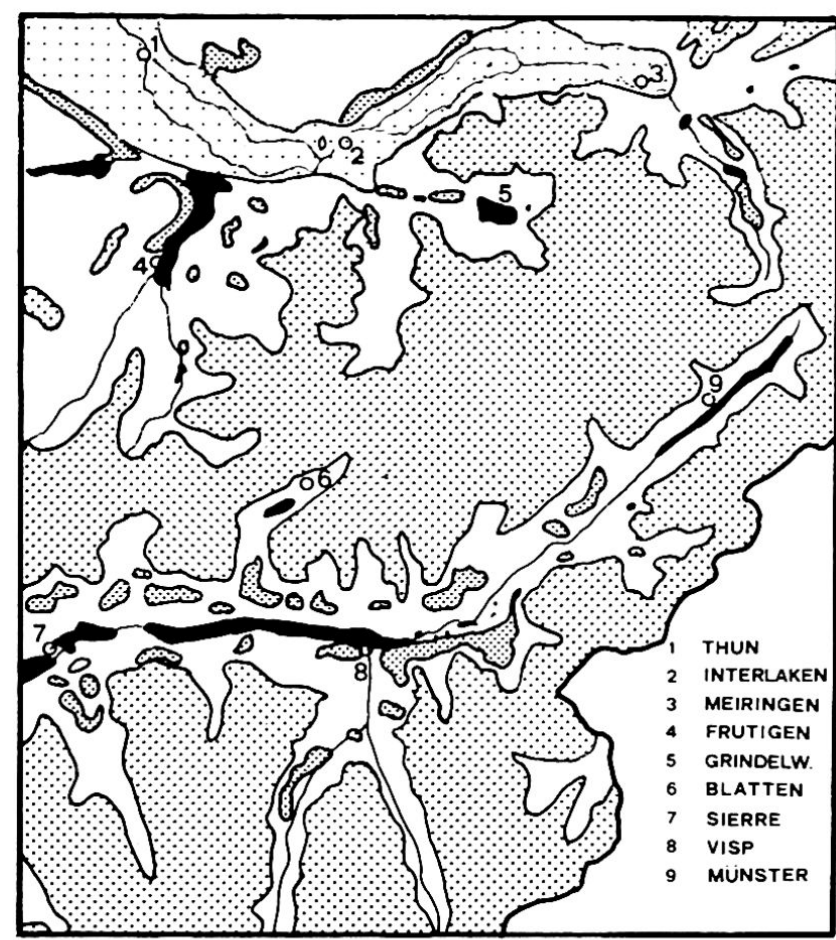

HCMM

10. 11.78

01.23 UT
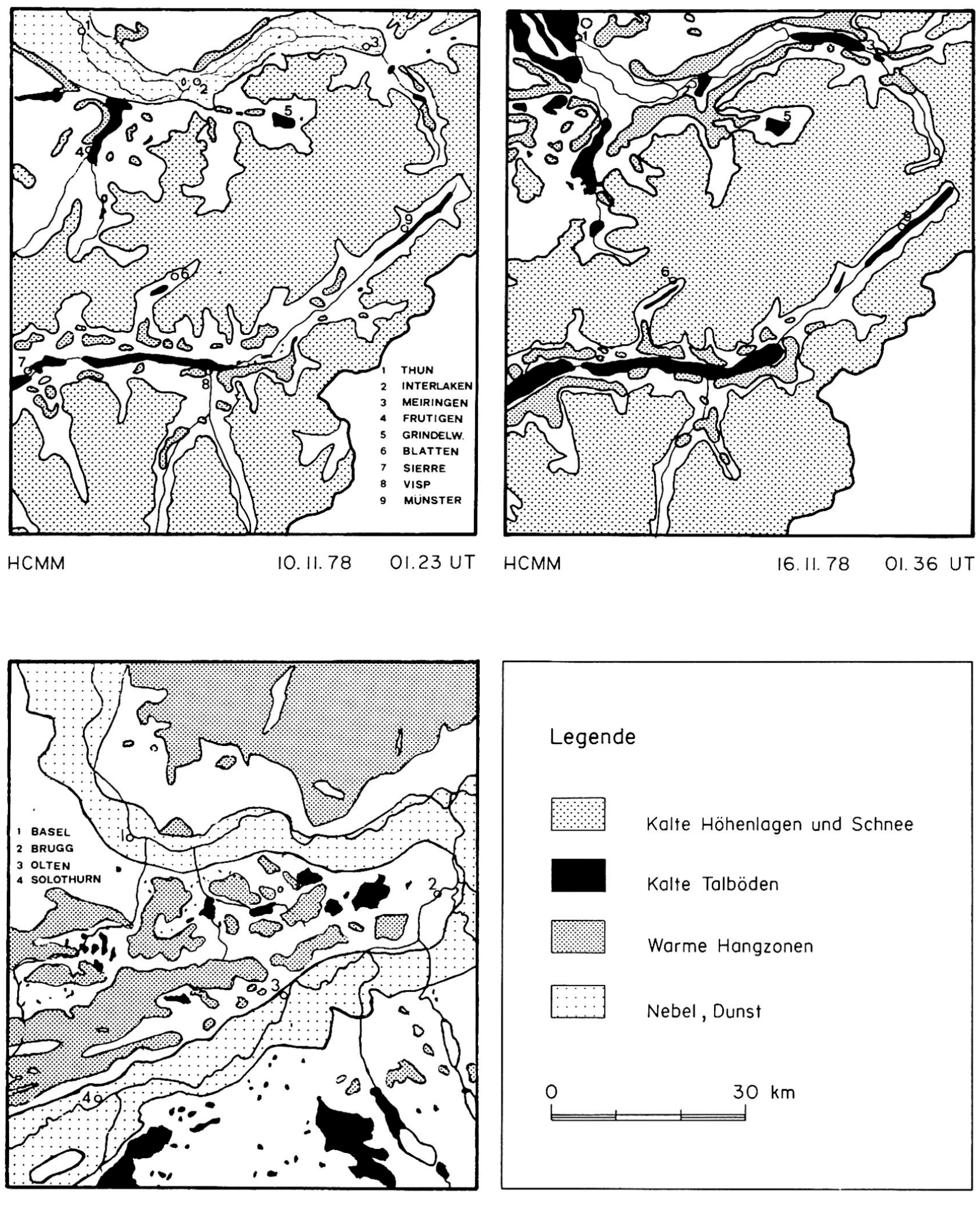

Legende

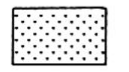

Kalte Höhenlagen und Schnee

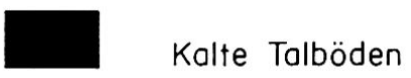

Warme Hangzonen

Nebel, Dunst

16. $11.78 \quad 01.36$ UT

$30 \mathrm{~km}$

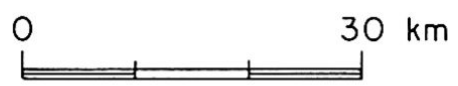

Fig. 6: Kartierung von Kaltgebieten (Talböden, Hochgebirge), warmen Hangzonen (z.T. Höheninversion) sowie Nebel- und Dunstfeldern aus HCMM-Thermalbildern. Der Vergleich von Nachtaufnahmen der Berner Alpen vom 10. und 16.11.1978 zeigt, daß Kaltgebiete (Bodeninversionen) streng topographisch gebunden sind, je nach Wetterlage verschieden stark ausgeprägt sein können. Für den Raum Jura-Schwarzwald (unteres Bild) kann eine Temperaturinversion auf ca. 800-900 m ü. M. festgestellt werden (NEJEDLY, in WINIGER, 1982b). 


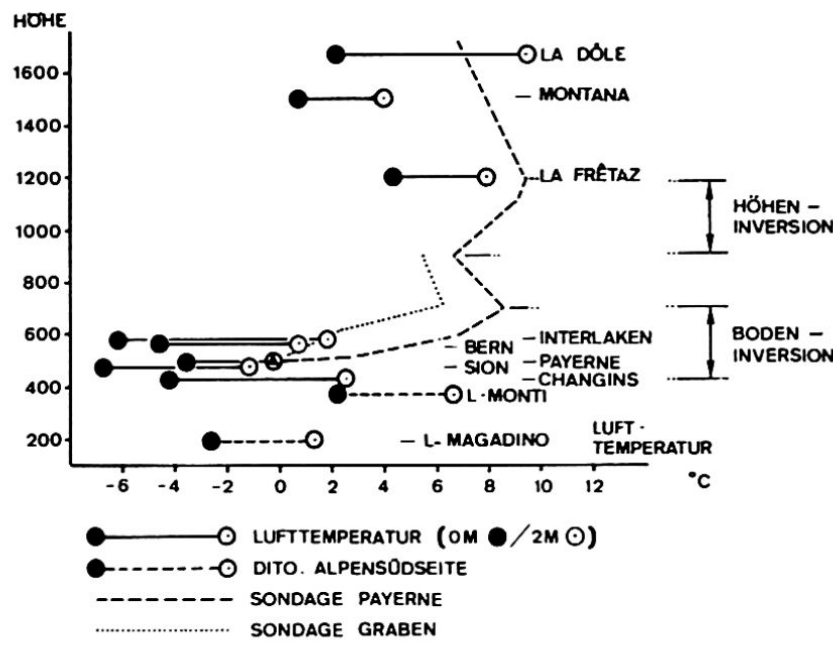

Fig. 7: Lufttemperaturwerte $(0 \mathrm{~m}, 2 \mathrm{~m}$, freie Atmosphäre) für Stationen auf unterschiedlicher Meereshöhe. 16.11.1978, 01.30 Uhr MEZ.

schnitte «Berner Oberland». Die in der Region Basel sich abzeichnende Höheninversion ist mit ihrer Untergrenze in das Höhenintervall $800-900 \mathrm{~m}$ ü. M. einzufügen. Der Bezug zu den effektiv gemessenen Lufttemperaturen ist in Fig. 7 enthalten: Im Mittel liegen die bodennahen Luftemperaturwerte um rund $5,5^{\circ} \mathrm{C}$ tiefer als die Hüttenwerte $(2 \mathrm{~m})$, nochmals also ein deutlicher Hinweis, daß Bodenoberflächen- und Lufttemperaturwerte beträchtlich voneinander abweichen können. Bemerkenswert ist, daß die Sondagenwerte (Payerne) die Inversionsuntergrenze auf der gleichen Höhenlage angeben wie die Bodentemperaturkartierung.

Als Schlußfolgerung ergibt sich, daß - zumindest in Nacht-Infrarot-Aufnahmen - Kaltluftgebiete sowie ausgeprägte Höheninversionen qualitativ abgrenzbar sind.

\section{Städtische Wärmeinseln}

Als Spezialfall der modifizierten natürlichen Energieund Strahlungsbilanz gilt die "Städtische Wärmeinsel». Sie führt dazu, daß die Lufttemperatur in überbauten Gebieten gegenüber dem Umland erhöht ist, im langjährigen Mittel und für schweizerische Verhältnisse um $1-3^{\circ} \mathrm{C}$, in Großstädten (z. B. Mailand) aber auch wesentlich höhere Beträge ausmachen kann. Zahlreiche radiometrische Messungen aus Flugzeugen zu allen Tages- und Jahreszeiten über der Stadt Basel ergaben eine mittlere Erhöhung der Bodentemperatur von rund $3^{\circ} \mathrm{C}$ gegenüber dem Umland, wobei der Temperaturunterschied an Sommertagen bis auf mehr als $12^{\circ} \mathrm{C}$ ansteigen kann. Der eigentliche Informationsgewinn der flächendeckenden Oberflä-

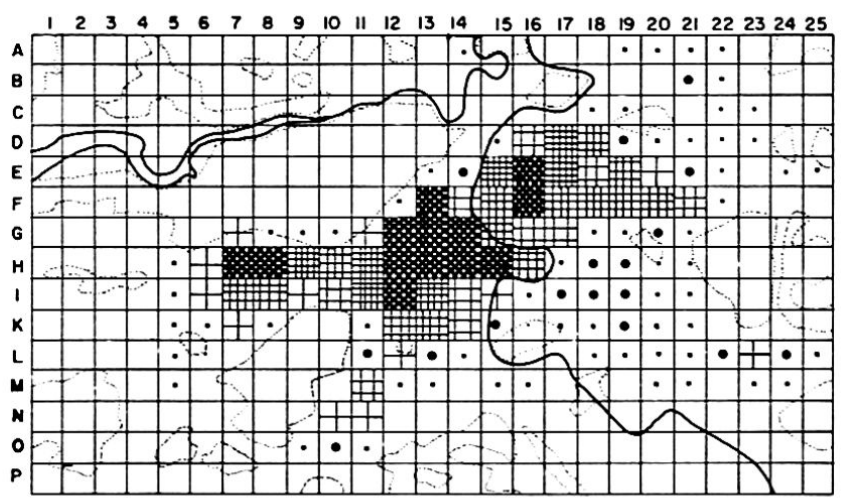

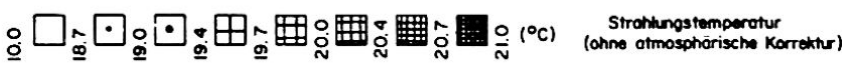

Fig. 8: Bodenoberflächentemperaturverteilung im Bereich der Stadt Bern, kartiert nach digitalen HCMM-Daten. Die Temperaturverteilung ist weitgehend abhängig vom Vegetationsanteil. 31.8.1979, 12.55 Uhr MEZ.

chentemperaturmessung aus Flugzeugen und Satelliten liegt aber in detaillierten Angaben über die räumliche Differenzierung des Temperaturfeldes. Als Beispiel ist in Fig. 8 die Temperaturgliederung der Stadt Bern aufgeführt. Obwohl in dieser Sommeraufnahme die thermischen Unterschiede zwischen Stadt und Land nicht eben groß ausfallen, ergibt sich doch eine gute Übereinstimmung mit der innerstädtischen Gliederung der Bebauungstypen: Siedlungsverdichtungen (Bern-West, Innenstadt, Breitenrainquartier) zeichnen sich durch höhere Temperaturwerte aus, während stark durchgrünte, locker bebaute Quartiere (Kirchenfeld) deutlich schwächer erwärmt werden. Vergleichsmessungen der Luftemperatur auf Meßfahrten im Stadtbereich ergeben von der Struktur her ein weitgehend identisches Bild (ABEGGLEN, 1982).

\section{Schlußfolgerungen}

Die hier skizzierten Auswertungen von HCMMThermalaufnahmen, ebenso wie die Ergebnisse anderer Autoren in vergleichbaren Untersuchungen (GossMANN, 1980), belegen die vielversprechenden Möglichkeiten der Satellitendatenauswertung im Rahmen geländeklimatischer Untersuchungen. Die Konzentration auf weitgehend wolkenfreie Wetterlagen oder auf Fälle mit Nebelbedeckung bedeutet insofern keine schwerwiegende Einschränkung, als dies in der Regel auch die geländeklimatisch am stärksten differenzierenden Wettersituationen sind. Die Heat Capacity Mapping Mission lieferte Daten optimaler räumlicher Auflösung zu geländeklimatisch idealen Überflugszeiten. 
Mit den Wettersatelliten der jüngsten Generation (TIROS-N, NOAA-6f) stehen nun aber auch operationelle Systeme zur Verfügung, die bei einer um den Faktor 2 reduzierten räumlichen Auflösung, dagegen einer zeitlich wesentlich dichteren Aufnahmerate (4-6 Aufnahmen pro Tag), digitale Information in 5 Spektralbereichen liefern, deren Brauchbarkeit sich in geländeklimatologischen Fragestellungen ebenfalls schon erwiesen hat. Im Zentrum der weiteren Bemühungen stehen folgende Schwerpunkte: 1. Qualitative und quantitative Deutung der multispektralen Information im Hinblick auf einzelne Klimaelemente. 2. Vereinfachung und Operationalisierung der Auswerteverfahren. 3. Optimale Kombination der verschiedensten Erhebungsmethoden (Satellit, Flugzeug, Meßfahrt, Fixstation). 4. Klimatologie der Nebel-, Dunstund Wolkenverbreitung, der Kaltluft- bzw. Inversionszonen. 5. Erarbeitung von Prozess- und Modellvorstellungen, Visualisierung ausgewählter atmosphärischer Vorgänge mit Hilfe von Satellitendaten.

\section{Literatur}

ABEGGLEN, R. (1982): Die städtische Wärmeinsel - Eine Literaturstudie und Städtetypisierung mit einem Beitrag zur Wärmeinsel von Bern. Diplomarbeit Geogr. Inst. Univ. Bern, $165 \mathrm{~S}$.

COENDET, M. (1979): Thermische Gliederung und lufthygienische Probleme im Talkessel von Grindelwald. Diplomarbeit Geogr. Inst. Univ. Bern, 113 S.

CLIMOD (1981): Möglichkeiten regionaler Klimaveränderungen durch menschliche Einwirkungen. Schlußbericht über das Projekt CLIMOD. Eidg. Kommission Meteorologie des schweizerischen Gebietes Hochrhein/Oberrhein, Bern.

GOSSMANN, H., LEHNER, M., STOCK, P. (1981): Wärmekarten des Ruhrgebietes - Satelliten-Thermalbilder der Heat Capacity Mapping Mission (HCMM). In: Geographische Rundschau, 33: 556-562.

GOSSMANN, H. (1980): A study of the relation between nighttime surface temperatures and land use, based on HCMM and LANDSAT images. TELLUS-Newsletter, No. 20, Ispra.

HAEFNER, H., ITTEN, K., WINIGER, M. (1980): Earth resources satellite application for planning purposes in Switzerland. In: Geographica Helvetica, 35(5): 71-76.

LICHTENEGGER, J. (1980): Landnutzungskartierungen mit multitemporalen LANDSAT-MSS-Daten. Diss. Univ. Zürich, JurisVerlag, Zürich.

NASA (1980): Heat Capacity Mapping Mission Users' Guide. Goddard Space Flight Center, Greenbelt, Md.

NEJEDLY, G. (1980): Probleme der geländeklimatologischen Auswertung von Satelliten-Infrarotaufnahmen. Diplomarbeit Geogr. Inst. Univ. Bern.
REINIGER, P. (1981): HCMM satellite data calibration and atmospheric corrections. TELLUS-Newsletter No. 25, Ispra.

SCHWAB, Z. (1981): Auswertung von digitalen Satellitenbilddaten - Ein Beitrag zum HCMM-Programm. Diplomarbeit, Geogr. Inst. Univ. Bern.

WANNER, H. (1982): Eine Detailanalyse der Durchlüftung in der Schweiz - Konzept und erste Resultate. Tag'ber. 17. Intern. Tag. f. Alpine Meteorologie, Berchtesgaden.

WANNER, H., KUNZ, S. (1982): Klimatologie der Nebel- und Kaltluftkörper im schweizerischen Alpenvorland mit Hilfe von Wettersatellitenbildern (in Vorb.).

WINIGER, M. (1974): Die raum-zeitliche Dynamik der Nebeldecke aus Boden- und Satellitenbeobachtungen. In: Informationen und Beiträge zur Klimatologie, 12: 24-29, Bern.

WINIGER, M. (1975): Topoclimatological and snowhydrological survey of Switzerland. Proposal of HCMM-Investigation submitted to NASA. Geogr. Inst. Univ. Bern.

WINIGER, M. (1982a): Klimatische Aspekte des Kernkraftwerkbaus (Studie CLIMOD). In: Geographische Rundschau, 34: 218-227.

WINIGER, M. (1982b): Topoclimatological Survey of Switzerland. Final report of HCMM-Investigation HCM-021. Prepared for NASA. Geogr. Inst. Univ. Bern.

WMO (1977): The role of satellites in WMO programmes in the 1980s. World Weather Watch Planning Report No. 36, WMONo. 494. Geneva. 